\title{
Changes In Fatty Acids Profiles After Three Weeks Of High-Fat Diet Feeding In Obesity-Prone Rats
}

\author{
Tzu-Wen Liu
}

\section{Dr. John P. Thyfault, Thesis Supervisor}

\begin{abstract}
High-fat diet (HFD) feeding is commonly used in rodents to induce obesity, but the effect of HFD on serum fatty acid profiles remains unclear. Changes in serum fatty acid profiles due to HFD may be a factor in lipotoxicity in various organs. Historically, in vitro studies have utilized individual non-esterified fatty acids (NEFA) to study lipid exposure in cultured cells, potentially ignoring the importance of fatty acid combinations that occur during obesity. OBJECTIVE: To characterize circulating fatty acids in obese-prone rats fed a HFD in both fasted and fed states to create a physiologically relevant fatty acid mixtures for cell culture studies. METHODS: Obesity prone rats were fed a HFD $(60 \% \mathrm{kcal}$ fat) or a control diet (10\%kcal fat) for 3 weeks; liver, portal and systemic blood samples were collected. Triglycerides (TG) and NEFA in the serum, TG, diglycerides (DG) and phospholipids (PL) in the liver were extracted and analyzed using gas chromatography. RESULTS: Both systemic and portal serum TG were $\sim 40 \%$ lower in HFD. Overall, the HFD induced greater proportion of polyunsaturated fatty acids (PUFA) compared to monounsaturated fatty acids (MUFA) in both TG and NEFA compared to the control diet. The elevations of PUFA were mostly attributed to the increased of n-6 PUFA, linoleic acid and arachidonic acid. CONCLUSION: HFD increased the n-6 PUFA to MUFA ratio in circulating fatty acid pools, thus a more physiologically relevant fatty acid mixture for in vitro studies may include increased linoleic acid and arachidonic acid relative to saturated fatty acids and MUFA.
\end{abstract}

